\title{
A TWO-DIMENSIONAL PARTICLE SIMULATION OF THE MAGNETOPAUSE CURRENT LAYER
}

\author{
J. Berchem and $H$. Okuda
}

\section{DSCLAMER}

This repor was prepared as an acoount of work sponsased by an agency of the United States Government. Neither the United States Government nor any agency thereof, nor any of their employees, makes any warranty. express or implied, of assumes any legal jiability or respengjbility for the accuracy, completeness, or usefulness of any information, apparatus. product, or process disclosed, or represents that its use would not infringe privately owned rights. Reference herein to any spesilic commercial product, process, or service by trade slame, trademark, manufacturer, or otherwise does not necessarily constitute or imply its endorsement, recommendation, or favoring by the Unitod States Government or any agency thereof. The views and opisions of authors expressed herein fo not necessanily state or refiect those of the United States Government or any agency thereot.

\section{Princeton Plasma Physics Laboratory \\ Princeton University}

Princeton. NJ 08543 
We have developed a $2^{\prime} / 2-D\left(x, y, v_{x}, v_{y}, v_{z}\right)$ electromagnetic code to study the formation and the stability of the magnetopause current layer. This code computes the trajectories of ion and electron farticles in their self-consistently generated electromagnetic field and an externally imposed 2-0 vacuum dipolar magnetic field. The results presented here are obtained for the simulation of the sclar windmagnetosphere interaction in the subsolar region of the equatorial plane.

We observe the self-consistent establishment of a current layer resulting from both diamagnetic drift and ExB drift due to the charge separation. The simulation results show that during the establishment of the current layer. its thickness is of the order of the hybrid gyroradius $p_{H}=\sqrt{\rho_{i} p_{e}}$ predicted by the Ferraro-Rosenbluth model. However. diagnostics indicate that the current sheet is subject to an instability which broadens the width of the current layer. Ripples with amplitudes of the order of the ion gyroradius appear at the interface between the field ard the particles. These pertubations are observed both on the electrostatic field and on the compressional component of the magnetic field. This instability has a frequency of the arder of the iccal ion cyclotron frequency. However, the modulation propagates in the same direction as the electron diamagnetic drift which indicates that the instability is not a classical gradient-driven instability. such as the lower hybrid or ion drift cyclatron instabilities. The nonlinear riase of the instability is characterized by the filamentation of the current layer which causes anomalous diffusion inside the central current sheet. 
Introduction

Recent controversies on the mechanism of the generation of flux transfer ovents (FTEs) has revived interast in the microstructure of the earth dayside ma yetopause and shown the need for a new theoretical effort to study its stability. We present here the results of $2^{1 / 2}$-dimensional electromagnetic particle simulations which relate to the formation and stability of the current layer.

Historically, the theoretical investigation of the solar wind interaction with the earth's magnetic field started with Chapman and Ferraro (1931). Assuming a perfectly conducting and unmagnetized solar stream, they showed the confinement of the dipole field by currents flowing in the front surface of the stream. This is the current layer that now we call the magnetopause. Although Hoyle (1949) and Alfven (1950) have aiready suggested the presence of an interplanetary magnetic field (IMF). early works only considered the prcblem of the equilititum between an unmagnetized plasma and a vacuum region of constant magnetic fie!d (Farraro. 1952; Rosenbluth. 1957: Dungey. 1958, 1961: Grad. 1961: Longmire, 1963: Sestero. 1965: see also reviews by Willis, 1971,1975 ).

With the start of spatial exploration, the Iirst dayside magnetic field profiles (Cahill and Amazeen, 1963) confirmed the presence of a magnetized sheath between the shock and the magnetopause. constituted by a highly turbulent subsonic and super-Alf venic plasma flow. Measurements also showed nonvanishing particle densities in a layer adjacent to the magnetospheric side of the discontinuity. These observaticns led to a second class of microscopic models of the magnetopause where the current sheet separates two magnetized plasmas. These magnetopause models are usually based on analytical solutions of Vlasov-Maxwell equations and differ mainly by the choice of initial velocity distribution functions, number $\mathrm{cl}_{1}$;omponents, and boundary conditions (see review by Lee and Kan, 1979). Ther? is no magnetic field component normal to the magnetopause surface. The boundary is impermeable to the solar wind flow. This type of magnetopause structure corresponds to the tangential discontinuity of the magnetohydrodynamics (MHO) description (Landau and Lipshitz. 1960).

A radically different type of magnetopause structure was introduced by Dungey's (1961) new developments on particle acceleration at magnetic neutral points (Giovanelit. 1946). Challenging Chapman and Ferraro's idea of a strict confinement of the geomagnetic fieid. his model suggests that a small fraction of the dayside earth's magnetic field lines can be connected to the interplanetary medium during periods o! 
southward IMF. As a consequence, instead of being a closed boundary, the magnetopase becomes open to the transfer of solar wind mass and momentum. Reconnection models (Petschek, 1964: Levy et al., 1964: Sonnerup, 1970: Yeh and Axford, 1970: Vasyliunas. $1975)$ predict the existence of a magnetic component normal to the surface of the discontinuity and high-speed flows. The dayside magnetopause is then of the rotational discontinuity type (Su and Sonnerup, 1968; Hudson. 1971: Sonnerup and Ledley, 1974; Lee and Kan. 1982; Swift and Lee. 1983). This steady-state MHD flow madel has provided the basis of the magnetopause paradigm that most of both experimenters and theoreticians have been pursuing during the past decade.

From the experimental point of view. the past twenty years of space exploration have permitted the accumulation of enough measurements to calculate typical plasma parameters at the dayside magnetopause (see reviews by Haerendel and Paschmann. 1982: Paschmann. 1984a; Elphic 1987). Magnetosheath plasma llow speeds ( $v_{f}$ ) vary from one to a few hundred $\mathrm{km} / \mathrm{s}$. These velocities are small compared to the sound speed. $V_{S}=200-400 \mathrm{~km} / \mathrm{s}$, but remain larger than the Alfven velocity $\left(V_{A}\right)$ which is of the order of $100 \mathrm{~km} / \mathrm{s}$ for average density and magnetic field $\left(n_{M S}=10 \mathrm{~cm}^{-3}\right.$. $\left.\mathrm{B}_{\mathrm{HS}}=15 \mathrm{nT}\right)$. The temperature $\left(T_{i}\right)$ of the bulk of the ion distribution varies between 5 and $10.10^{B}{ }^{\circ}$. so the magnetosheath ion gyroradius $\left(\rho_{i}\right)$ is of the order of $100 \mathrm{~km}$. The ratio of the ion to electron temperatures $\left(T_{i} / T_{e}\right)$ is around 10 and the temperature anisotropy $\left(T_{\perp} / T_{\|}\right)$lluctuates between 1 and 2 in frunt or the magnetopause.

More recently. the ISEE multispacecraft missions allowed substantial progress in the investigation of magnetospheric boundaries, especially the magnetopause. Indeed, for the rirst time, most of the instruments were able to provide enough adequate time resolution measurements to study magnetopause phenomena, and simultaneous in-situ measurements at different locations were used to measure macroscopic features of the magnetopause boundary, such as the thickness $(400$ to $1600 \mathrm{~km}$ ) and the velocity (10 to $80 \mathrm{~km} / \mathrm{s}$ ) of the current layer (Berchem and Russell, 1982). Of course. a great deal of effort has been devoted to analyzing correlative plasma and field measurements in order to identify dayside magnetic reconnection. The observations of some of the predicted signatures have to some extent supported the steady-state model (see reviews by Paschmann. 1978. 1984b; Russell. 1984 a,b; Sonnerup, 1984).

A new and important magnetopause feature emerged with the identification of temporally unsteady and spatially very localized reconnection processes, observed both in the polar cusps (Haerendel et al.. 1978) and in the equatorial region (Russell and Elphic. 1978. 1979). These observations. which have been called flux transfer events (FTES). have considerably altered the theoretical approach of the study of the microstructure and stability of the magnetopause. Indeed, although the equilibrium of the magnetopause was questioned in the iate 1950's, those studies only focused on the 
stability of the magnetospheric flank and tail boundaries. Eviatar and Wolf (1968) using quasilinear theory. suggested that the two-stream ion-cyclotron instability could scatter ions at a distance comparable to their gyroradius within a few gyroperiods. They calculated that for a 100-km-thick boundary. the transfer of momentum would be adequate to drive the magnetospheric convection postulated by Axford and Hines (1961). Attention was also drawn by the problematical confinement of the geomagnetic tail (Lerche, 1967: Parker, 1967a,b). Nevertheless, Alpers (1971). Su and Sonnerup (1971) demonstrated that an equilibrium does exist even in a region where the solar wind particles have a bulk velocity tangential to the geomagnetic field.

Before the FTEs became closely associated with the stability of the dayside magnetopause. Gary and Eastman (1979) suggested that the lower hubrid drift instability (Davidson and Krall. 1977) could account for the bursty low Irequency $(<1 \mathrm{OOHz})$ electric and magnetic fluctuations observed by Gurnett et al. (1979). However. they also noted that the mode is probably not excited by thick boundary layers, but should be driven only by smaller scale density gradients. The possible occurrence of another instability, the tearing mode (Furth et al., 1963). was also suggested from both laboratory terrella experiments (Dubinin et al., 1980) and space observations at the Earth and Jupiter's magnetopauses (Greenly and Sonnerup. 1981). In response to these observations. linear theory of the guide field tearing instability (Laval et al., 1966; Coppi et al., 1966; Galeev and Zeleny. 1978) was derived for high-\$ plasmas (Ouest and Coroniti. 19810) in order to study the tearing at the dayside magnetopause (Quest and Coroniti. 1981a). It was shown that the linear grow th rate of the instability is a sensitive function of the IMF orientation. magnetopause thickness, and electron number density. Quest and Coroniti (1981 a,b) also emphasized that tearing should involve a two-dimensional wave spectrum $\left(k_{y} \times 0\right)$ tangential to the magnetopause and that tearing-reconnection should be spatially inhomogeneous and temporally unsteady. Thus, it is natural that the tearing instability has been proposed to explain FTE generation (Lee and Fu, 1985: Kan, 1988; La Belle-Hamer et al., 1988). Nevertheless, since the initial Russell and Elphic's (1978) FTE model of intermittent reconnection of individual flux tubes, many other mechanisms have also been advanced. They are based on stochastic percolation of the magnetic field through the magnetically destroyed surfaces (Galeev et al., 1986) or sporadic multiple reconnection processes (Ding et al., 1986; Sato et al., 1986; Shi et al.. 1988; Sholer, 1988).

In this paper we examine the fundamental interaction between the subsonic magnetosheath flow and the magnetospheric fieli. We think that it is neccessary to achieve a better understanding of the basic physical process before pursuing more sophisticated magnetopause models by including other fundamental ingredients such as 
the magnetosheath field. the presence of energetic particles of magnetospheric origin. or the coupling with the ionosphere. We thus re-examine the classical

Ferraro-Rosenbluth problem of the equilibrium between a plasma and a vacuum magnetic field. In fact, the kinetic treatment or this interaction has not been addressed in a self-consistent manner since the ore-dimensional study by Hasegawa and okuda (1967). Most of the past studies of the tangential discontinuity structure of the magnetopause assume an ad hoc initial Harris (1962) type of equilibrium. On the other hand. recent nonlinear numerical models. such as the ones proposed for the generation of FTEs. use MHD or hybrid codes and thus ignore electron Landau damping and electrostatic effects. However, as several studies (Ouest and Coroniti. $1981 \mathrm{~b}$; Lembege and Pellat, 1982; Swift, 1986) have demonstrated, the electron dynamics is primordial in the physics of current sheets.

To study the formation and the stability of the subsolar magnetopause current layer. we have thus developed a two-dimensional simulation code which computes this trajectories of both ion and electron particles in their self-consistently generated electromagnetic rields and an externally imposed magnetic field. Our model consists of an unmagnetized subsonic super-Alfvenic plasma flow injected transversely to a two-dimensional vacuum dipolar magnetic field.

\section{Simulation model and computational method}

Figure 1 shows the geometry of our simulation system. The $L_{x} \times L_{y}$ domain represents the subsolar region of the equatorial plane in which we follow the trajectories of solar wind particles. The ion and electron particles are injected irom the left. so the right boundary delineates the magnetospheric side of our system. since for numerical convenience we $f i x$ the origin $(x=0)$ of our system of coordinates at the injection boundary, our reference irame is oricnted oppositely to conventional geccentric systems of coordinates. The $x$ axis points towards the Earth. the $y$ axis is directed from dusk to dawn. and the $z$ axis points northward.

To model the Earth's magnetic field, we use a two-dimensional magnetic dipolar field generated by two infinite parallel wires aligned with the $y$ direction. We can then assume that all the system is uniform and periodic along the $y$ axis. The non-zero components of the field are given by:

$$
\begin{aligned}
& \theta_{x}=2 \theta_{0} \frac{-x z}{\left(x^{2}+z^{2}\right)^{2}} \\
& B_{z}=B_{0} \frac{x^{2}-z^{2}}{\left(x^{2}+z^{2}\right)^{2}}
\end{aligned}
$$


where $X=x_{D}-x_{\text {, and }} x_{D}$ is the location of the dipole axis. However. In order to keep a significant $B_{z}$ variation within the limits of cur system without imposing a too strong field on the left boundary. we have to position the axis of the dipole relatively close to the left boundary $\left(X_{D}=4 L_{X}\right)$. In return such a model leads to an excessive field line curvature in the immediate vicinity of the simulation plane. In reality. in the near equatorial region of the subsolar magnetopause. the radius of the field curvature is mueh larger than the thickness of the current layer and $B_{z}>>B_{X}$. To study the limit of a small curvature of the field line, we assume that the field is independent of $z$ $\left(B_{x}=0 ; B_{z}=B_{0} / x^{2}\right)$. The initial magnetic field strength at $z=0$ is such that before compression, the ratio of the average electron gyrofrequency to the electron plesma frequency ( $\omega_{c e} / \omega_{p e}$ ) is equal to 0.5 at $x=0$ and to 3.125 at $x=L_{x}$. Furthermore. at the start of the simulation, $B_{0}$ is ramped linearly over the first 50 electron gyroperiods and then held constant. This "quiet start" technique allows us to apply smoothly and self-consistently the boundary conditions that we describe later on. We introduce a Iinite computational grid on the $x-y$ plane, with a grid size $\Delta$, such that $L_{x}=200 \Delta$ and $L_{y}=128 \Delta$. and define a dimensionless system of units by chonsing $\Delta$ and $\omega_{\text {pe }}$ to be the spatial and time units of our system. We define $c$ the speed of light such that the collisionless skin depth $c /$ upe $_{\text {is }}$ equal to $5 \Delta$.

The calculation is then initialized by loading a solar wind particle reservoir ad jacent to the left boundary of our simulation system. We use finite-size ion ard electron particles to reduce the collision frequency and we jistribute them uniformly to obtain a number density $\left(n_{0}\right)$ equal to 4 per each $\Delta^{2}$ gris cell of tho reservoir. We also initialize the particle components of velocity $v_{x}, v_{y}$, and $v_{z}$ from a Maxwellian velociry distribution function generator such that the electron thermal velocity $v_{T e}$ is equal to $\Delta \omega_{\text {pe. }}$. The grid size $\Delta$ than becomes identical to the Debye length $\left(\lambda_{0}\right)$ of our system. Note that since we assume no spatial change along the 2 direction ano derine three velocity components, our model is $21 / 2$-dimensional. Ion to electron mass $\left(M_{i} / m_{e}\right)$ and temperature $\left(T_{i} / T_{e}\right)$ ratios used are equal to 100 and 9 , respectively. After initialization, a constant drift velecity $v_{d x}=0.25 \Delta \omega_{p e}$ is then added to the $x$-componest of the solar wind particles to simulato the subsonic. super-Alfvenic magnetosheath flow. The sound speed is slightly grester than the ion thermal speed $\left(v_{T i}\right)$ which is equal to $0.3 \Delta / \omega_{\text {pe }}$. The initia' Alfven speed before comi ression $\left(v_{A}=c\left(\omega_{c e} / \omega_{p e}\right)\left(m_{e} / M_{i}\right)^{1 / 2}\right)$ varies irom $0.25 \Delta \omega_{p s}$ at $x=0$ to $1.5 \Delta \omega_{p e}$ at $x=x_{L}$.

We then start the iterative loop of the simulation by calculating the charge $p$ and the current densities $J$ produced by all particles entered in the system by using the linear interpolation scheme (Birdsall and Fuss. 1969: Marse ano Nielson. 1969):

$$
\begin{aligned}
& \rho(x, y)=\sum a_{j} s_{j}(x, y) . \\
& J(x, y)=\sum q_{j} v_{j} s_{j}(x, y) .
\end{aligned}
$$


where $s_{j}(x, y)=\frac{1}{2 \pi \Delta^{2}} e^{-\frac{\left[\left(x-x_{j}\right)^{2}+\left(y-y_{j}\right)^{2}\right]}{2 \Delta^{2}}}$ is a Gaussian-shaped factor for the finte-size particies used to redice the collision frequency. Once $J$ is calculated, we break down the transwerse $\left(J_{t}\right)$ and longitudina: $\left(J_{l}\right)$ parts of the current density by assuming that the longitudinal current $d_{l}$ is derived from a potential $\left(J_{l}=-\nabla \psi\right)$. and proceed to the integration of the time-dependent Maxwell's equations:

$$
\begin{aligned}
& \nabla \times E_{t}=-\frac{1}{c} \cdot \frac{\partial \theta}{\partial t} \\
& \nabla \times B=\frac{1}{c} \frac{\partial E_{t}}{\partial t} \cdot \frac{4 \pi}{c} s_{t} .
\end{aligned}
$$

To be consistent with our assump?ion of an unmagnetized plasme flow in the magnetosheath. We impose the condition that the $B_{y} \cdot B_{z}$ and $E_{y}$. $E_{z}$ components vanish on the left and right boundaries, respectively, and proceed to the integration in time of these equations by using a second order accurate time-centered method (Langdon et al.. 1976). We complete the set of electromagnetic fields by calculating the electrostatic field which is purely longitudinal:

$$
\nabla \varepsilon_{s}=4 \pi \rho \text {. }
$$

We solve that equation by assuming no boundary perturbations. sc the electrostatic potential $\phi$ vanishes $\left(E_{s}=-\nabla \phi\right)$ on the left and right boundaries. After a Fourier transform of all the quantities along the $y$ direction, the corresponding Poisson's second order differential equation is given by:

$$
\frac{d^{2} \phi\left(x, k_{y}\right)}{d x^{2}}-k_{y}^{2} \phi\left(x, x_{y}\right)=4 \pi \rho\left(x, k_{y}\right)
$$

which is easily solved by a triangularizarion method.

Finally. once the total $E(x, y)$ and $B(x, y)$ fields are completely determinad, we use exact dynamics to advance the particles by solving the equations of motion:

$$
m_{j} \frac{d v_{j}}{d t}=a_{j}\left(E_{t o t}+\frac{1}{c}-v_{j} \times \theta_{t o t}\right)
$$

using Buneman's (1968) scheme.

The code has been tested for numerical dissipation by loading the entire system of a magnetospheric plasmia without injecting the solar wind. A run over $500 / \omega_{\text {pe }}$ shows that the total energy is conserved within a few percent. 
Figure 2 shows a composite display of a few diagnostics of our simulation at time $t=140 / \omega_{p e}$. On the lelt-hand column, we plotted as a furction of the $x$ position, the electron number Jensity $n_{e}$ (a). the $E_{x}$ component of the sum of the ambipolar electrostatic field and the electric perturbation (b), the current density $J_{y}$ (c). and the $\mathrm{B}_{2}$ component of the magnetic field (d). Here and after, all the one-dimensional plots that we show have been obtained by averaging each quantity both in time in order to eliminate high frequency noise, and spatially over the $y$-direction to show only the $k_{y}=0$ mode. On the right-hand column of the same figure, we display instantaneous two-dimensional scatter plots of the electrons and ions which show their locations $[(e)$ and ( 1 ) respectively] in the $x-y$ plane and their phase space distributions $[(g)$ and (h) respectively] in the velocity phase space $x-v_{x}$. These diagnostics show the self-establishment of a current layer between the magnetosheath plasma and the magnetospheric field. The longitudinal electric field $E_{x}(b)$, mainly due to the charge separation between ions and electrons. generates a negative $(y<0)$ transver se electron current by a $C E \times B / B^{2}$ drift. An additional but smaller current is generated by the diamagnetic drift $J_{D}=-\nabla p \times B / B^{2}$ due to the strong density gradient seen in Fig. $2(a)$. Notice that the nonzero electric field and the density depletion observed at the injection boundary are just local effects due to the electron gyration at the edge of our simulation box and do not affect the rest of the system. Both ExB and diamagnetic currents flow in the same direction and contribute to the formation of the sharp current layer. $J_{y}$ shown in Fig. $2(c)$, which compresses the magnetic field $B_{Z}$ (d) and produces the magnetic field gradient characteristic of the magnetopause. Nevertheless. as the ion velocity distribution in Fig. $2(\mathrm{~h})$ shows. only a fow ions have a negative $v_{x}$ velocity. This means that the bulix of the particles have not reached the point where the particle pressure balances the magnetic pressure. Although a few particles have already penetrated deeply in the region of higher magnetic field. other diagnostics indicate that these ions also have negative $v_{y}$ suprathermal velocities and thus have larger ion gyroradii than the bulk of the particles. Note that the electron $v_{x}$ velocity distribution shown in Fig. $2(g)$ is already symmetrical because of the trapping by the local magnetic field.

As time progresses, the beam advances in the system, pushing and compressing the magnetic field, until ions get reflected. Naturally, the location of the reflection should be determined by the pressure balance condition. Since we suppose that the flow has already been shocked before the injection, when writing the balance condition, both terms for the dynamic and the thermal pressures have to be retained; 
thus $2 n M_{i} v_{d x}{ }^{2}+K T_{i} \approx B_{z}{ }^{2} ; 8 \pi$, where $K$ is the usual boltzmann's constant. The fact that the beam velocity $v_{d x}=0.25 \Delta \omega_{\text {pe }}$ is very close to the ion thermal speed $v_{T i}=0.3 \Delta \omega_{\text {pe }}$ has further implications: we can use this to simplify the previous balance equation and rewrite it in terms of the ratio $\omega_{\mathrm{ce}} / \omega_{\text {pe. }}$. Firstly. only about half of the particles penetrate into the system. This is readily seen in Fig. 2(a) which shows that the beam has a number density equal to 2 instead of the initial 4 in the partıcle reservoir. Since we use the full Maxwellian velocity distribution funct ions to define the simulation plasma parameters. we can eliminate from the plasma pressure terms the factor 2 which takes into account the jump in density due to the specular reflection of the particles. Secondly. since both velocities are rlose, we can estimate that both dynamic and thermal pressure contributions are roughly equal, so in first approximation we have $2 n M_{i} v_{d x}{ }^{2}=B_{z}{ }^{2} / 8 \pi$. This allows us to rewrite tne pressure balance condition as $\omega_{c e} / \omega_{p e}=2 \sqrt{M_{i} / m_{e}} v_{d x} / c$. With our set of initial values, this relation implies that the pressure balance is satisfied where $\omega_{c e} / \omega_{p e}=1$. Taking into account our normalization factors. this ratio corresponds to $\theta_{z} / B_{0}=5$, so we expect the location of the reflexion point to be around half of the system length $x_{R}=100 \Delta$.

Figure 3 displays the same diagnostics as in Fig. 2. but at a later time. $t=440 / \omega_{\text {pe }}$. As expected, we now observe the start of the reflection of the bulk of the particles. This is seen on Fig. 3(a), which shows that the beam density increases significantly to almost 3 particles per cell. and in Fig. 3(h). where it is readily seen that the ion $v_{x}$ velocity distribution is almost symmetrical. We also note that the current layer ( $3-d)$ advances to $x=80 \Delta$, sweeping the magnetic field. It turns out that the reflection point is closer to the loft than we expected. This is due to the compression of the field. Never'heiess, the actual ratio $\mathrm{B}_{2} / \mathrm{B}_{0}$ agrees with the prediction or the pressure balance condition. Another interesting feature is seen in the current lager profile in Fig. 3(c). Although its peak amplitude is slightly sinaller, the width of the current layer is substantially larger than the one observed at $t=140 / \omega_{\text {pe. }}$. We will comment on the structure inside the layer later on. Examining the two-dimensional scatter plots of tho particle $x-y$ locations. Figs. $3(e)$ and $3(f)$. we notice that this broadening correspends to the development of an instability at the interface of the plasma and the field. Naturally, the modulation appears clearer for the electrons (e) than for the ions ( $f$ ) because of their smaller gyroradii. Before discussing the results of the spectral analysis of this instability. let us examine the time history of the current sheet formation and the development of the insiability.

To follow the dynamics of the formation of the current layer we have compiled in Figs. $4(a)$ and $4(b)$ a series of diagnostics taken from iime $t=100 / \omega_{\text {pe }}$ to time

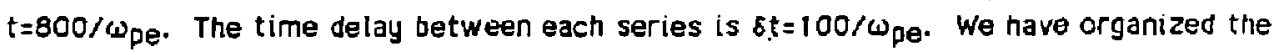
results in four columns. From left to right. we display two-dimensional plots of the 
electron $x-y$ locations, and one-dimensional plots of the electron density $n_{e}$. the current density $J_{y}$. and the magneric field $B_{z}$, versus the $x$ locatror: Inspection of the scatter plots of the electron $x-y$ locations (left column) shows a phase shift of the instability and indicates that we can distinguish 3 stages in the evolution of the interaction between the plasma and the magnetic field: firstly. ihe establishment of the current layer, seccondiy, the lineur growth of the instability. and inally, the nonlinear development.

From the start of the simulation to around time $t=200 / \omega_{F e}$. particles are pushing the field at a speed close to the injection velccity. As we see in Fig. 2 , only a few particles are already reflected back 35 the sligl.t density increase indicates. This phase corresponds to the seli-establishment of the cul ient layer. During this first stage the current iayer has an almost constant thickness of about $17 \Delta$. which is much smaller tian the approximately $40 \Delta$ ion gyroradius measured at the peak of the current density. We define the thickne:s of she curren: layer by measuring the width of the peak of the $J_{y}$ component at one-nall of its value. Ts sompare the observed thickness to the classical results of the Ferraro-Rosenbluth model. we have to calculate the average hybrid gyroradius $\rho_{H}=\sqrt{\rho_{i} \rho_{e}}$. which becomes $\rho_{H}=p_{i} \sqrt{30}$, using thermal velocities to calculate with ion and electron gyroradii. since at orte-half or the peak the observed $\rho_{i}$ varies batween $140 \Delta$ to $30 \Delta$, we obtain an average hibrid gyroradius of $15.5 \Delta$. which is very close to the thickness obsarved.

At the time $t=300 / \omega_{\text {pe }}$ the bump observed in the density profile indicates that more particles are reflected. However. as we know from Fig. 3, the bulk of the beam has not yet penetrated far enough for the particle pressure to balance the magnetic field pressure. so the beam is still progressing but has slowed down to almost half of the injection velocity. The current layer thickness is now at least of the order of one ion gyroradius. We also notice the presence of very small ripples on the scatter plois of the electrons (lert column). which indicates the growth of an iustability. This marks the beginning of the second stage which lasts until time $t=600 / \omega_{p e}$. This pericd of time corresponds to the linear phase of the instability. Note that since the instability started growing before the bulk of incoming ions start reflecting. its frequency and growth rate should be then comparable to the ion gyrofrequency. This will be discussed in a more quantitative manner when feporting the spectral analysis of the instability. Nevertheless, we can note that the maximum amplitude from the crest to the trough of the modulation is of the order of one ion gyroradius, so the lager thickens substantially to almost two ion gyroradii.

Finally. from time $600 / \omega_{\text {pe }}$ to the end of the similation [Fig. $4(\mathrm{~b})$ ]. we observe the development of the quasilinear ang highly nonlinear phases of the instability. The plots of the electron locations show first the steepening of the modulation, and then. 
star ing from time $t=700 / \omega_{p e}$ the formation of eddies. Looking at the current density profiles $J_{y} / J_{0}$. it is aiso apparent that the layer has considerably spread out while the initial peak has been reduced to half of its value observed during the linear phase of the instauility. This is due to the progressive pirch of the central current sheet which ultimately leads to the filamentation of the current layer at time

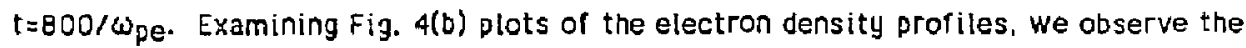
formation of a small plateau which gradually evolves as the instability becomes highly nonlinear. This change in the density profile is very similar to the one associated to the nonlinear evolution of the collisionless drift-wave instability. Lee and oxuda (1976) showed tiat as the instability develops, the waves grow in amplitude, causing quasilinear diffusion in density. The change in the density profile then alters the local dispersions and becomes the dominant stabilization mechanism of the instability. Unfortunately for us, this anomalous transport coincides with the end of the simulation when we run out of particles in the reservoir, so it is difficult to verify that the diffusion saturates the instability. However. the resulting local density plateau turns off the diamagnetic currents which cause the local pinch observed in the current layer proriles.

To characterize the instability. we have carried out several Fourier analyses of the electromagnetic and electrostatic fluctuations. For various $x$-locations, we analyzed the first five modes along the $y$ direction of the system. Naturally. the strongest fluctuation is observed on the $B_{z}$ component for $k_{y}=0$, because it corresponds to the compression of the dipole field. Figure 5 shows the analysis of the magnetic perturbation $\mathrm{SB}_{Z}$ measured into the reflection region at location $x=80 \Delta$. The top panel displays time series of $\delta \mathrm{B}_{2} / \mathrm{B}_{\mathrm{O}}$. where $\mathrm{B}_{\mathrm{O}}$ is a normalization factor. One can see tnat $\delta B_{z} / B_{0}$ first reaches a maximum around time $t=300 / \omega_{p e}$ and then decreases to become negative around time $t=600 / \omega_{p e}$. This variation is simply due to the advance of the current layer and appears as a peak at zero frequency in the power spectrum shown in the lowest panel of Fig. 5. Looking back at the time history. We aiso notice a superimposed modulation with a period around $64 \omega_{p e t}$. This is the signature of kinetic Alfven waves asscciated with the compression of the magnetic field. As we calculated earlier. $\omega_{\mathrm{ce}} / \omega_{\mathrm{pe}} \approx 1$ at the reflection point, so the local $\mathrm{A}_{\mathrm{l}}$ lfven velocity $\left(V_{A} \approx c\left(\omega_{c e} / \omega_{p e}\right)\left(m_{e} / M_{i}\right)^{1 / 2}\right)$ is around $0.5 \Delta / \omega_{p e}$. Using the magnetosonic dispersion relation $\omega= \pm k_{y} V_{A}$ and the kinetic approximation $k_{y}=2 \pi / \rho_{i}$ with $\rho_{i}=30 \Delta$, we find that $\omega \approx \pm 0.1$. Which is consistent with the small twin peaks observed in the power spectrum.

For $\mathrm{K}_{\mathrm{y}}=0$, significant turbulence is observed on the electrostatic and compressional magnetic $B_{z}$ components. Figures $6 a$ and $6 b$ display the time history and the power spectrum of the alectrostatic potential $\Phi$ and $\delta B z$, respectively, measured 
at $x=80 \Delta$ for the dominant mode $k_{y}=4 \pi / L_{y}$. For both we observe a strong peak at a very low frequency $\left(\omega=-0.018 \omega_{p e}\right)$ which is close to the local ion cyclotron frequency $\left(\omega_{c} i=-0.01 \omega_{\text {pe }}\right.$ at the reflection point). After normalization, calculation shows that both fluctuations are of the same crder. To check this frequency against the phase shift that we noticed earlier in Fig. 4. we show in Fig. 7 a sequence of the scatter plots of the electron locations (left column) and the corresponding contours of the electrostatic potential (right column). We start at the bottom of the figure with plots measured at time $t=340 / \omega_{\text {pe }}$ and display each series after a time delay $\delta t=40 / \omega_{\text {pe }}$ until time $t=500 / \omega_{\text {pe. }}$. We observe clear the motion of the structures in the $y>0$ direction. which means that the modulation propagates in the direction of the electron diamagnetic drift. The structures move about $10 \Delta$ between each snapshot. which corresponds to a phase velocity about $0.25 \Delta \omega_{\text {pe }}$. Using the dominant mode $k_{y}=4 \pi / L_{y}$. this transiates into a real frequency $\omega=-0.025 \omega$ pe. Taking into account that the Nyquist frequency $\omega \approx 0.008 \omega_{\text {pe }}$, wo find that this estimate is very close to the frequency obtained from the power spectrum analysis.

To explain the growth of an instability due to the presence of strong density and magnetic field gradients, it is natural to think of classical gradient-driven instabilities such as the lower hybrid drift or the drift cyclotron instabilities. However, for $k_{x}=0$ these instabilities propagate in the direction of the ion diamagnetic Jrift (Ka:monstev, 1965). Furthermore, the observed frequency is much lower than the lower hybrid. The occurrence of such instabilities may then be excluded, so we do not uispose of ready analytic predictions to compare to our simulation results. To this aim. we have initiated the theoretical analysis of the linear phase. Encouraging preliminary results (Hasegawa. 1988; personal communication) indicate that the instability can be explained by the combined effect of both magnetic und density gradients. The instability condition obtained with a local approximation can be expressed as $4 v_{0} / k \omega_{c i}>1$, where $k=(1 / \theta) \partial \theta / \partial x-(1 / n) \partial n / \partial x>0$ and $v_{0}$ is the electron drift velocity. The characteristic frequency obtained is then $\omega=-\omega_{c i} k_{y} / k$. Considering that the gradient scale length is of the order of 2 ion gyroradii or about $60 \Delta$. the previous relation gives us a frequency $\omega \approx-1.5 \omega_{c i}$ for the dominant mode $k_{\mathrm{y}}=4 \pi / L_{\mathrm{y}}$. This result shows that both directions of the phase velocity and frequency range observed in the simulation are reproduced by the analytical calculation. Details of the cerivation will be reported elsewhere. 


\section{Discussion and Conclusions}

In this paper we have examined the fundamental interaction detween the magnetosheach flow and the magnetospheric field using a $21 / 2$-dimensional simulation. We deliberately attacked this problem with a very simple model to be abie to carry out a full kiretic electromagnetic simulation with a realistic computer time budget. We chose a 2-dimensional magnetic dipole with the field direction perpendicular to the simulation plane and iimposed periodic conditions along the y direction. of course these assumptions limit the extent of our study to the equatorial region of the subsolar magnetopause. In this model. the magntopause is flat and particles cannot be convected out of the system as they do along the flanks of the real magnetopause. It is thus difficult to assess the relevance of our results to experimental observations. However, several comments can be drawn from the results presented above.

Firstly, one thing that stands very clearly is that because of the growth of the instability. the current layer appears to be thicker than the hybrid gyroradius predicted by the Ferraro-Rosenbiuth model. In the past this discrepancy was resolved by arguing that in the presence of a magnetospheric plasma and a cold plasma trapped in the current layer, the ambipolar electric field should be short circuited by the ionospheric plasma being pulled along the field lines (Parker, 1967a). Once the polarization lield neutralized, the ions should be able to penetrate fully in the magnetospheric field, and the thickness of the current layer should be just the average ion gyroradius. Ulterior sophistications of our model that include magnetospheric plasma and the effects of the ionospheric conductivity should clarify the role of field-aligned currents at the dayside magnetopause. A further broadening of the rurrent sheet due to the ionospheric coupling would certainly help us to use our simulation results to explain the magnetopause thickness measurements. Indeed. Berchem and Russell (1982) showed that the magnetopause current layer is thicker than about 5 ion gyroradii and that the thickening appears to be much better when ordered by the dipole magnetic latitude than any other parameters, so that the current layer is thinnest near the equator. Since the maximum thickness observed in our simutation is less than 3 ion gyroradti, it is difficult to use our present results to explain their observations. Since our simulation is limited to the equatorial plane, a further study of the instability dependence on the field line curvature is needed to resolve whether the instability can account lor the latitudinal variation of the observations. 
Secondly. another important point shown by our simulation results is the occurrence of a microinstability which has a frequency of the order of the local ion cyclotion frequency and propagates in the direction of the electron drift. Such fluctuations are indeed consistent with the ion cyclotron waves observed at the magnetopause (Fairfield, 1976; Anderson et al., 1982) and the bursty low frequency electrostatic and magnetic fluctuations reported by Gurnett et a!. (1979).

Finally. the nonlinear evolution of the instability towards the filamentation of the current sheet by anomalous diffusion is somewhat surprising. However. we have to keep in mind that spacecraft experiments measure fields, not currents. Examination of high resolution magnetic field profiles across the dayside magnetopause very often reveals the presence of small structures inside the magnetic field gradient. Although it has always been difficult to resolve the ambiguity between spatial and tempora! variations from the analysis of times series. ISEE dual magnetometer ouservations (Russell and Elohic. 1978) have shown several cases where spatial structures are clearly identified inside the magnetopause current layer. Examination of high resolution particle data, when they become available. should resolve whether these structures are caused by quasilinear diffusion.

Belore concluding. we wish to remark that it is difficult to evaluate the role of the initial boundary conditions in the stabilization of the instability. However, earlier calculations (Berchem and Okuda, 198B) carried out with a much smaller system (128×64) led us to the same results as reported hero. This seems to indicate that the walls are far enough away to affect significantly the saturation process. Definite resolution of this question will be possible by improving our code to load more particles and use an even bigger sustem than the $(200 \times 128)$ grid user here.

In summary, we have developed a $2^{i} / 2-D\left(x, y, v_{x}, v_{y}, v_{z}\right)$ electromagnetic code to study the formation and the stability of the magnetopause current layer. Our mode! consists of an unmagnetized subsonic super-Alfvenic plasma flow injected transversely to a two-dimensional vacuum dipolar magnetic field. The results presented are obtained for the simulation of the solar wind-magnetosphere interaction in the subsolar region of the equatoriai plane.

1. The simulation results show that during the establishment of the current layer, its thickness is of the order of the hybrid gyroradius as predicted by the Ferraro-Rosenbluth model. However, diagnostics indicate that the current sheet is subject to an instability which broadens the width of the current layer. Ripoles with an amplitude of the order of the ion gyroradius appear at the interface between the field and the particles.

2. Perturbations are observed both on the electrostatic field and on the compressional component of the magnetic field. The instability has a frequency of the 
order of the local ion cyclotron frequency. However. the modulation propagates in the same direction as the electron diamagnetic orift, which indicates that the instability is not a classical gradient-driven instability such as the lower hybrid dritt or ion drift cyclotron instabilities.

3. The nonlinear phase of the instability is characterized by the filamentation of the current layer which causes anomalous diffusion inside the central current sheet.

Acknowledgments. The authors like to acknowledge useful discussion with $H$. Abe on the electromagnetic simulation. and with A. Hasegawa on the analysis of the linear stability of the current layer. This research is supported by the National Science Foundation Grant No ATM-85-12512, the San Diego Supercomputer Center, and the U.S. Department of Energy Contract No DE-ACO2-76CHO3073. 
Alfven, H.. Cosmical Electrodynamics, Oxford University Press, London, 1950.

Alpers, W., On the equilibrium of an exact charge neutral magnetopause. Astrophys. Space Sci., 11, 471, 1971.

Anderson, R.R., C.C. Harvey, M.M. Hoppe, B.T. Tsurutani, T.E. Eastman, and J. Etcheto. Plasma waves near the magnetopause. J. Geophys. Res.. 87. 2087. 1982.

Axford. W.I., and C.O. Hines. A unifying theory of high-latitude geophysical phenomena and geomagnetic storms. Can. J. Phys., 39. 1433. 1961.

Berchem. J., and C.T. Russell. The thickness of the magnetopausa current layer: ISEE 1 and 2 observations. J. Geophys. Res., 87, $2108,1982$.

Berchem, J., and H. Okuda. Numerical simulation of plasma turbulence and anomalous diffusion at the magnetopause current layer. in Nonlinear phenomena in Vlasov plasmas, edited by F. Doveil and D. Gresillon. PMI Ecole Polytechnique. Paris, 1988.

Birdsall, C.K., and D. Fuss. Clouds-in-clouds, clouds-in-cells physics for many body simulation, J. Comput. Phys., 3, 494, 1969.

Buneman, 0 .. Fast numerical procedures for computer experiments on relativistic plasmas. in Relativistic Plasmas, edited by O. Buneman and W. Pardo, p. 205. Benjamin. New York. 1968.

Cahill, L.J., and P.G. Amazeen. The boundary of the geomagnetic field. J. Geophys. Res.. $68,1835,1963$.

Chapman, S., and V.C.A. Ferraro. A new theory of magnetic storms, Terr. Mag., 36, 171, 1931.

Coppi. B., G. Laval and R. Pellat, Dynamics of the geomagnetic tail, Phys. Rev. Lett., $16,1207,1966$.

Davidson, R.C., and N.A. Krall, Anomalous transport in high-temperature plasmas with applications to solenoidal fusion systems. Nucl. Fusion. 17, 1313, 1977.

Ding. D.Q., L.C. Lee and Z.F. Fu, Multiple $X$ line reconnection 3. a particle simulation of flux transfer events. J. Geophys. Res., 91, 13384. 1986.

Dubinin. E.M.. I.M. Podgornyl and Y.N. Potanin, Structure of the magnetic field at the boundary of the magratosphere: analysis of a simulation experiment. Translated from Kosmicheski Issledovaniya, 18, 99, 1980.

Dungey. J.W.. Cosmic Electrodynamics. p. 137. Cambridge University Press. London. 1958.

Dungey, J.W., The steady state of the Chapman-Ferraro problem in two dimensions, J. Geophys. Res., 68, 1043, 1961 a.

Dungey. J.W., Interplanetary magnetic ileld and the auroral zones, Phys. Rev. Lett., 6. 47. $1961 \mathrm{~b}$.

Elphic. R.C., The bow shock and the magnetopause, Rev. of Geophys., 25, 3, 510, 1987.

Eviatar. A.. and R.A. Wolf. Transfer processes in the magnetopause. J. Geophys. Res.. 73. 5561, 1968.

Fairfield. O.H.. Waves in the vicinity of the magnetopause, in Magnetospheric Particles and fields, edited by B. McCormac, p. 67, D. Reidel, Dordrecht, Holland, 1976. 
Ferraro, V.C.A., On the theory of the first phase of a geomagnetic storm: a new illustrative on an idealized (plane not cylindrical) model field distribution. J. Geophys. Res., 57, 15, 1952.

Furth. H.P.. J. Killeen and M.N. Rosenbluth, Finite-resistivity instabilities of a sheet pinch. Phys. Fluids, 6, 459, 1963.

Galeev. A.A., and L.M. Zeleny, Magnetic reconnection in a space plasma, in Theoretical and Computational Plasma Physics, p. 93, International Atomic Energy Agency. Vienna, Austria, 1978.

Galeev. A.A., M.M. Kuznetsova and L.M. Zeleny. Magnetopause stability threshold for patchy reconnection, Space Sci. Rev., 44, 1, 1986.

Gary. S.P., and T.E. Eastman. The lower drift instability at the magnetopause, J. Geophys. Res., 84. 1979.

Giovanelli, R.G.. A theory of chromospheric llares. Nature, 158, 81, 1946.

Grad. H., Boundary layer between a plasma and a magnetic field. Phys. Fluids, 4, 1366. 1961.

Greenly. J.B., and B.U.O. Sonnerup. Tearing at the magnetopause, J. Geophys. Res., 86. $1305,1981$.

Gurnett, D.A., R.R. Anderson, B.T. Tsurutani, E.J. Smith. G. Paschmann, G. Haerendel. S.J. Bame. and C.T. Russell, Plasma wave turbulence at the magnetopause: observations Irom ISEE 1 and 2. J. Geophys. Res.. 84, 7043. 1979.

Haerendel. G.. and G. Paschmann. Interaction of the solar wind with the dayside magnetosphere, in Magnetospheric Plasma Physics, edited by A. Nishida, p. 49. D. Reidel. Dordrecht. Holland, 1982.

Haerendel. G., G. Paschmann. N. Sckopke. H. Rosembauer and P.C. Hedgecock. The front side boundary layer of the magnetosphere and the problem of reconnection, $J$. Geophys. Res., B3, $3195,1978$.

Harris. E.G.. On a plasma sheath separating regions of oppositelj directed magnetic rield. Nuovo Cimento, 23, $115,1962$.

Hasegawa. A., and H. Okuda. Computer experiment on Ferraro-Rosenbluth problem, J. Flys. Soc. Japan, 23, 906, 1967.

Hoyle. A., Some recent researches in solar physics, Cambridge University Press, London. 1949.

Hudson, P.D., Rotational discontinuities in an anisotropic plasma. Planet. Space Sci.. $21,475,1971$.

Kadomtsey, B.B., Plasma turbulence. p 95. Academic. New York. 1965.

Kan. I.R.. A theory of patchy and intermittent reconnections for magnetospheric Ilux transfer events. J. Geophys. Res., 93. 5613. 1988.

La Belle-Hamer. Z.F. Fu and L.C. Lee. A mechanism for patchy reconnection at the dayside magnetopause, Geophys, Res. Lett., 15. 152, 1988.

Landau. L.D. and E.M. Lifshitz, Electrodynamics of continuous media, p. 224, Pergamon Press. New York, 1960.

Langdon. A.B., and B.F. Lasinski, Electromagnetic and relativistic plasma simulation models, in Methods of Computational Physics, 16, edited by B. Alder. S. Fernbach. M. Rotenberg and J. Killeen. p. 327. Academic. New York. 1976.

Lava:. G., R. Pellat and M. Vuillemin, Instabilités électromagnetiques des plasmas sans collisions, in Plasma Physics and Controlled Fusion, vol. 2, p. 259. International 
Atomic Energy Agency, Vienna, Austria, 1966.

Lee, L.C., and J.R. Kan. A unified kinetic model of the tangential magnetopause structure, J. Gecphys. Res., 84, 6417, 1979.

Lee. L.C., and J.R. Kan. Structure of the magnetopause rotational discontinuity. J. Geophys. Res.. 87, 139, 1982.

Lee, L.C., and Z.F. Fu, A theory of a magnetic flux transfer at the earth's magnetopause, Geophys. Rev. Lett.. 12, 105.1985.

Lee. W.W.. and H. OKuda, Anomalous transport and stabilization of collisionless Arift-wave instabilities, Phys. Rev. Lett., 15, 870, 1976.

Lembege, B., and R. Pellat. Stability of a thick two-dimensional quasineutral sheet. Phys. Fluids, 22. 1995.1982.

Lerctie, 1 . On the boundary layer between a warm, streaming plasma and a confined magnetic rield., J. Gecphys. Res., 72. 5295. 1967.

Levy. R.H., H.E. Petschek, and G.L. Siscoe. Aerodynamic aspect of the magnetospheric HIOW. AlAA J.. 2, 2065, 1964.

Longmire, C.L., Elementary plasma physics, p. SO, Interscience Publishers. Wiley. New York, 1963.

Morse, R.L., and C.W. Nielson, Numerical simulation of a warm two beam plasma, Phys. Fluids. 12. 2418.1969.

Nicholson. R.B.. Solution of the Vlasev equation for a plasma in a external!y uniform magnetic field. Phys. Fluids. 6. 1581. 1963.

Parker. E.N., Confinement of a magnetic field by a beam of ions, J. Geophys. Res., 72. 2315, 1967 a.

Parker. E.N.. Small-scale nonequilibrium of the magnetopause and its consequences, J. Geophys. Res., 72. 4365, $1967 \mathrm{~b}$.

Paschmann, G.. Plasma structure of the magnetopause and boundary layer. in Magnetospheric Boundary Layers, edited by B. Battrick. p. 25. Rep. ESA SP-148. Paris, France, 1979.

Paschmann. G.. The earth's magnetopause, in Achievements of the International Magnetospheric Study. compiled by B. Battrick and E. Rolfe. p. 53, P.ep. ESA SP-217, Noordwijk. The Netherlands, $1984 a$.

Paschmann. G., Plasma and particle observations at the magnetopause: implication for reconnection, in Magnetic Reconnection in Space and Laboratory Plasmas, edited by E.G. Hones, p. 114, AGU, Washington, D.C., 19846.

Petschei. H.E.. Magnetic field anninilation. in AAS-NASA Symposium on the physics of sular flares, edited by W.N. Hess. pp. 425, Rep. NASA SP-50, Greenbelt, MD, 1964.

Quest. K.B., and F.V. Coroniti. Tearing at the dayside magnetopause. J. Geophys. Res.. 86. 3289, $1981 \mathrm{a}$.

Quest. K.B.. and F.V. Coroniti. Linear theory of tearing in a high-beta plasma. J. Geophys. Res., 86. 3299, 1981 b.

Rosenbluth. M.N.. Dynamics of a pinched gas, in Magnetohydrodynamics. edited by R.K.M. Landshoff. p. 57. Stanford University Press. California, 1957.

Aussell, C.T. The structure and dynamics of the magnetosphere: Progress during the IMS, in Achievements of the International Magnetospheric Study, compiled by $B$. Battrick and E. Rolfe, p. 67, Rep. ESA SP-217. Noordwijk, The Netherlands, 1984a.

Russell, C.T.. Feconnection at the earth's magnetopause: magnetic field observation and 
flux transfer events. in Magnetic Reconnection in Space and Laboratory Plasmas. edited by E.G. Hones, p. 124. AGU, Washington, D.C.. 1984b.

Russell, C.T., and R.C. Elphic, Initial ISEE magnetometer results: magnetopause observations. Space Sci. Rev.. 22, 681, 1978.

Fussell, C.T., and R.C. Elphic. 15EE observation of flux transfer events at the day magnetopause, Geophys. Res. Lett., 6, 33. 1979.

Sato, T., T. Shimada, M. Tanaka, T. Hayashi, and K. Watanabe, Formation of field-twisting flux tubes on the magnetcpause and solar wind particle entry in the magnetosphere, Geophys. Res. Lett., 13. 801. 1986.

Scholer. M.. Magnetic flux transfer at the magnetopause based on a single $X$ line bursty reconnection, Geophys. Res. Lett.. 15, 291, 1988.

Sestero. A.. Charge separation effects in the Ferraro-Rosenbluth cold plasma sheath model, Phys. Fluids, B, 739.

Shi, Y., C.C. Wu and L.C. Lee. A study of multiple $X$ line reconnection at the cayside magnetopause. Geophys. Res. Lett., 15, 295, 1988.

Sonnerup, B.U.O., Magnetic fieic reconnection in a highly conducting incompressible lluit. J. Plasma Phys., 4. 161. 1970.

Sonnerup. B.U.O.. Magnetic field reconnection at the magnetopause: an overview. in Magnetic Heconnection in Space and Laboratory Plasmas, edited oy E.G. Hones. D. 92. AGU. Washington, O.C.. 1984.

Sonnerup, B.U.O., and B.G. Ledley. Magnetopause rotational forms, J. Geophys. Ros., 74. 4309, 1974.

Su, S.Y., and B.U.O. Sonnerup. First order orbit thecry of rotational discontinuity. Phys. Fluids, 11, 851, 1960.

Su, S.Y.. and B.U.O. Sonnerup. On the equilibrium of the magnetopause current layer, $J$. Geophys. Res., 76. 5181, 1971.

Swift. D.W.. The effect of the ionosphere on the grow th of tearing mode instabilities in the magnetotail. J. Geophys. Res., 91, 219.1986.

Swift. D.W.. and L.C. Lee. Rotational discontinuity and the structure of the magnetopause, J. Geophys. Res., 88, 111.1983.

Vasyliunas. V.M.. Theoretical models of magnetic field line merging, Rev. Geophys. Space Phys., 13. 303, 1975.

Willis, D.M., Structure of the magnetopause, Rev. Geophys. Space Phys., 9, 953, 1971.

Willis. O.M., The microstructure of the magnetopause, Geophys. J. Ray. Astron. Soc., 41, 355. 1975.

Yeh. T., and W.l. Axford. On the reconnection of magnetic field lines in conducting fluids, J. Plasma Phys., 4, 207, 1970. 
Figure 1: Geometry of the simulation model. The $x$ axis points towards earth, the $y$ axis is directed from dusk to dawn, and the $z$ axis points northward. The $L_{x} \times L_{y}$ domain represents the subsolar region of the equaiorial plane in which we follow the trajectories of solar wind particles. The ion and electron particles are injected from the left boundary $(x=0)$ transversely to a 20 -dipalar magnetic field. We assume that all the quantities are periodic along the $y$ axis.

Figure 2: Composite display of several diagnostics of the simulation at time $t=180 / \omega_{\text {pe. }}$ On the left-hand column, we plotted as a function of the $x$ position: (a) the electron number density $n_{e}(b)$ the component $E_{x}$ of the total electric field, (c) the current density $J_{y}$. and (d) the magnetic fields $B_{z}$. On the right-hand column we display two-dimensional scatter plots of the electron (e) and ion (f) positions, and respectively $(g)$ and $(h)$ their phase space distribution in the velncity phase space $x-v_{x}$.

Figure 3: Composite display of the same diagnostics as in Fig. 2 but for time $t=440 / \omega_{p e}$

Figure 4 (a): Dynamics of the formation of the current layer. From left to right. One-dimensional plois of the magnetic firld $B_{z}$. the current density $J_{y}$. the electron density $n_{e}$, and the electron locations in the $x-y$ plane, taken from time

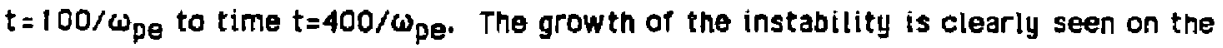
left column.

Figure 4(b): Dynamics of the formation of the current layer (continued). We use the same diagnostics as figure $4(a)$ from time $t=500 / \omega_{p e}$ to time $t=800 / \omega_{p e}$. This illustrates the quasilinear and the highly nonlinear phases of the instability.

Figure 5: Time history and power spectrum of the $\delta B z$ component of the magnetic field measured at $x=80 \Delta$ for mode $k_{y}=0$. On the upper panel, $\delta B_{z}\left(x_{z} k_{y}\right)$ is plotted as a function of time. The peak observed about the zero frequency range is due to the motion of the current layer during the compression of the magnetic field. The twin peaks observed at $\omega \approx-0.1 \omega_{p e}$ are the signatures of compressional kirietic Alfven waves. 
Figure 6a: Time history and power spectrum of the electrostatic potential $\phi$ measured at $x=80 \Delta$ for mode $k_{y}=4 \pi / L_{y}$. On the upper panel, both real and imaginary parts of $\phi\left(x, k_{y}\right)$ are plotted as a function of time. The strong peak observed in the very low frequency range $\omega \approx-0,018 \omega$ pe is due to the instability. The small twin peaks obser:ed at $\omega \approx-0.1 \omega_{\text {pe }}$ are the signatures of compressional kinetic Alf ven waves.

Figure 6b: Time history and power spectrum of the $8 B z$ component of the magnetic field measured at $x=80 \Delta$ for mode $k_{y}=4 \pi / L_{y}$. On the upper panel. both real and imaginary parts of $\delta \mathrm{B}_{z}\left(x, k_{y}\right)$ are plotted as a function of time. The strong peak observed in the very low frequency range $\omega=-0.018 \omega_{0 e}$ is due to the instability. The small twin peaks, almost unobservable at $\omega=-0.1 \omega_{\text {pe }}$, are the signatures of compressional kinetic Alfven waves.

Figure $7:$ Scatter plots of the electron $x-y$ location (left-hand column) and contour plots of the electrostatic potential (right-han.t column) show the modulation propagates ir the same direction as the electron diamagnetic drift at a phase velocity around $0.25 \Delta \omega_{\text {pe }}$ 


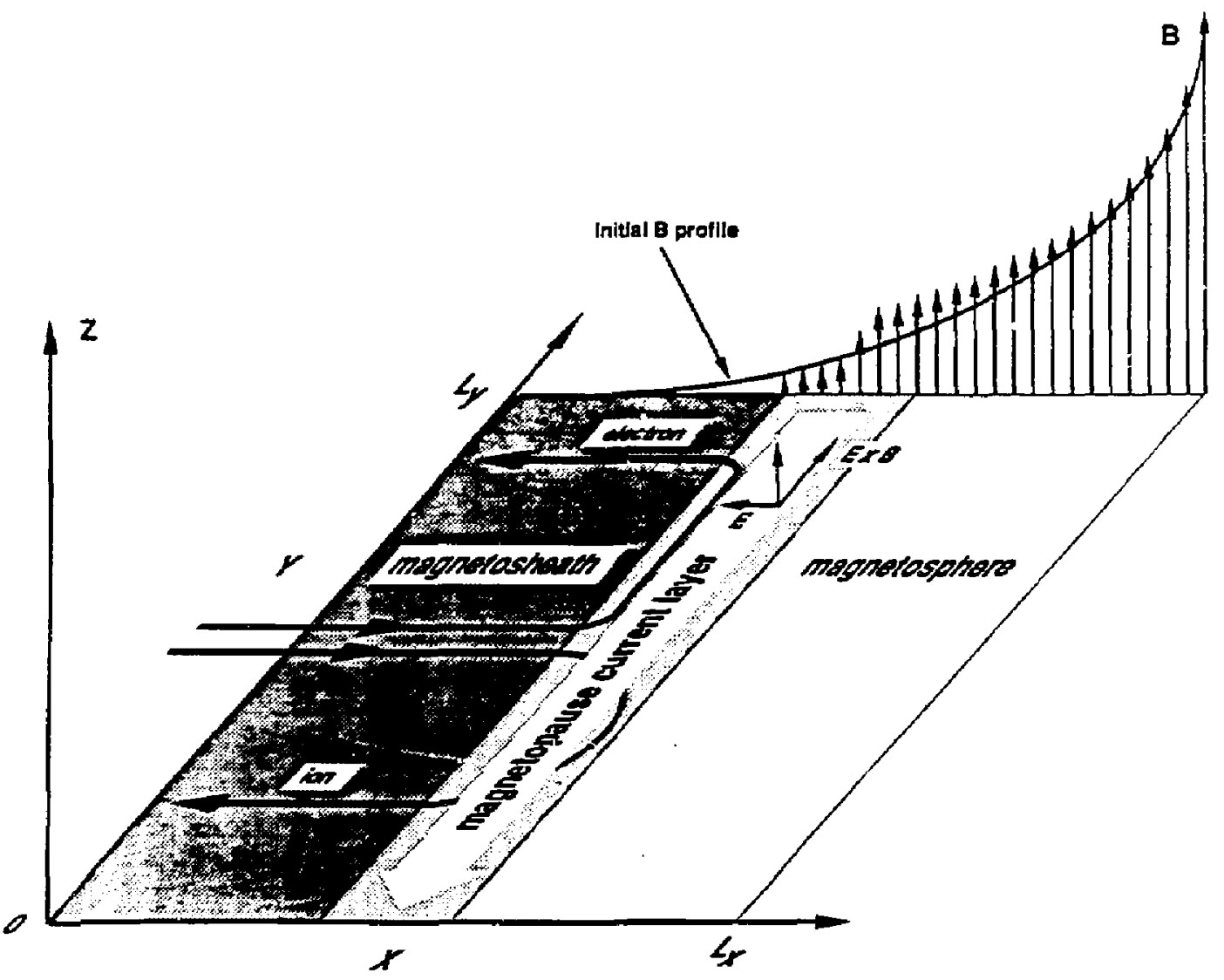

Figure 1: Simulation model 

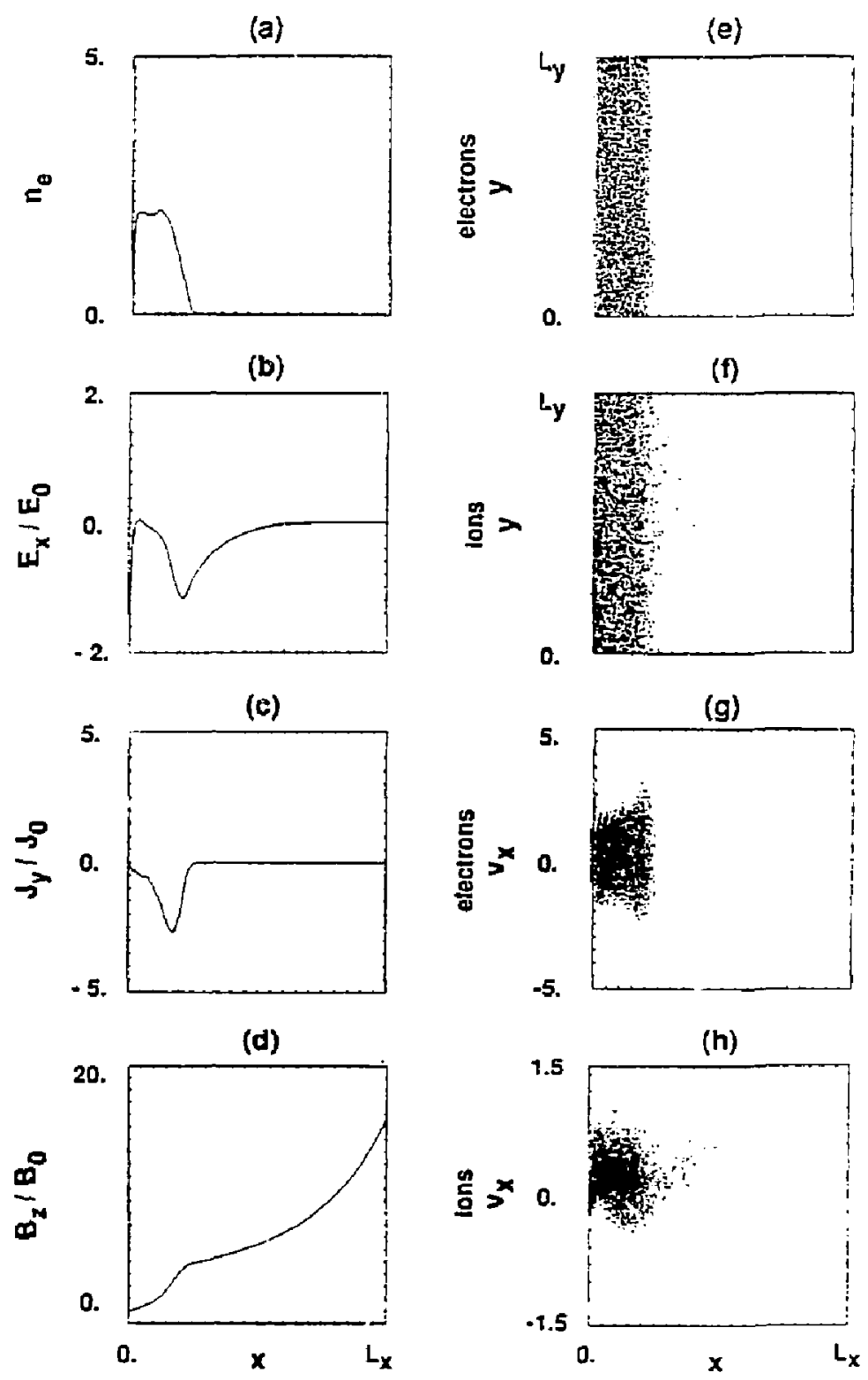

Figure 2: $t=: 40 / \omega \mathrm{pe}$ 


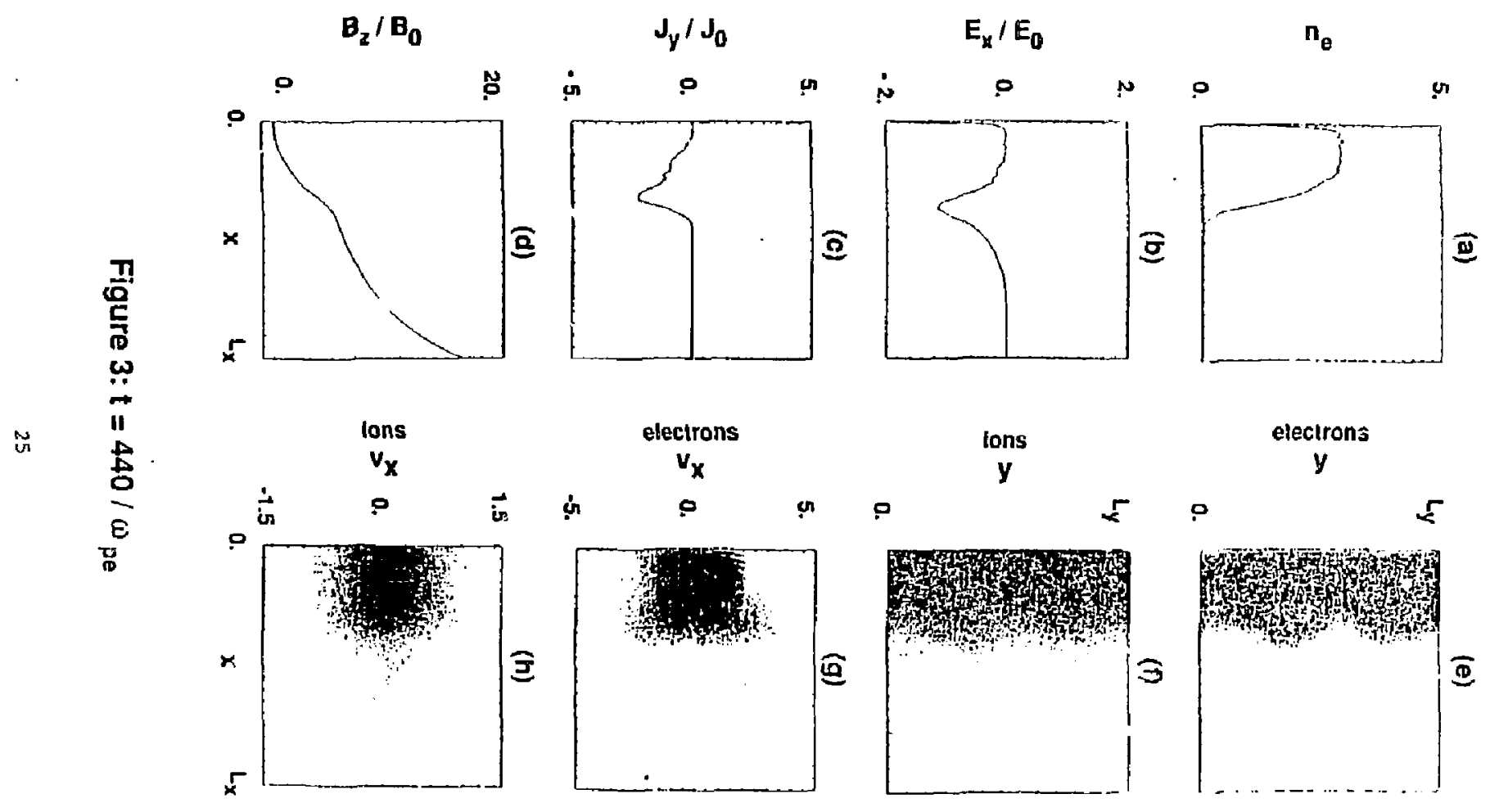




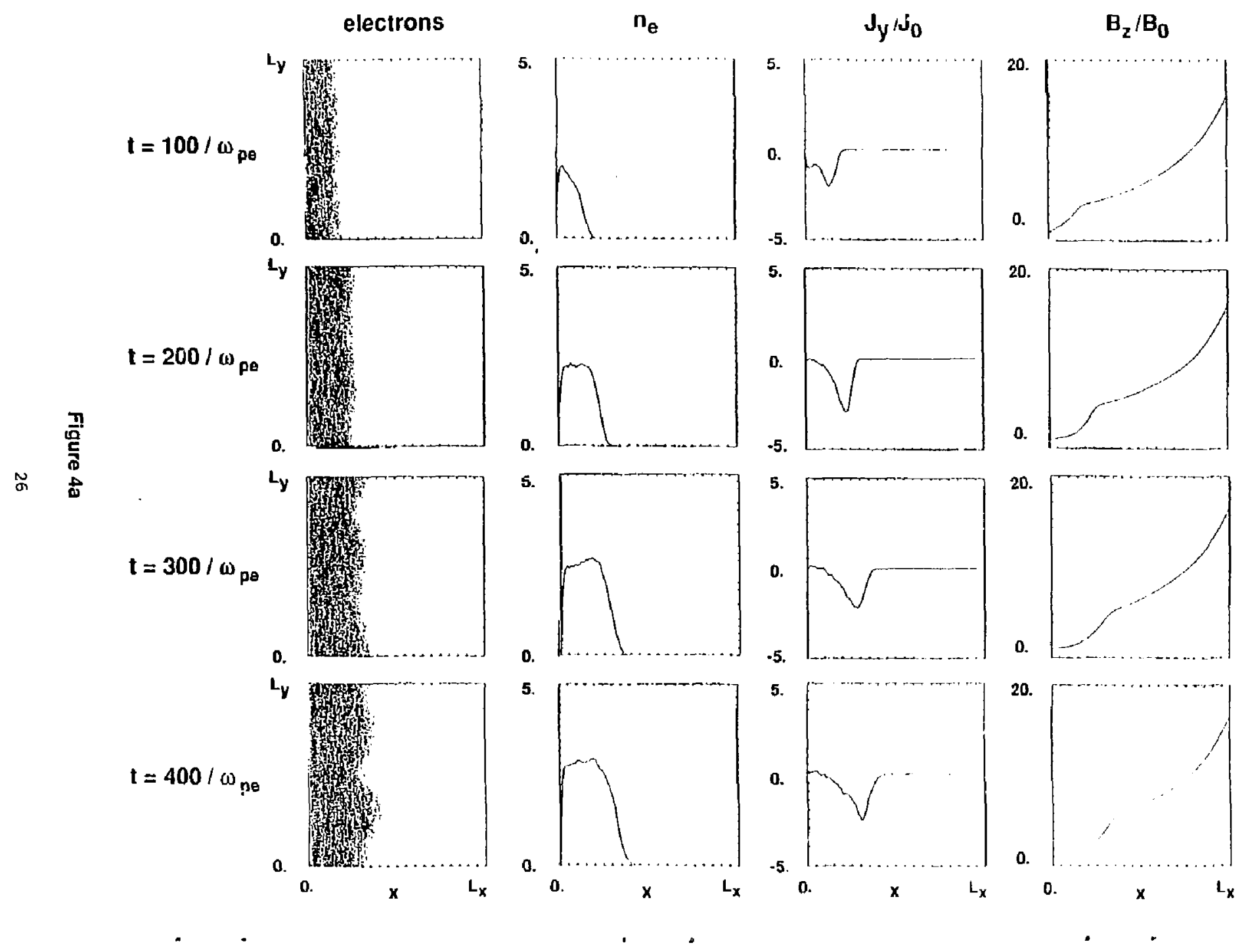



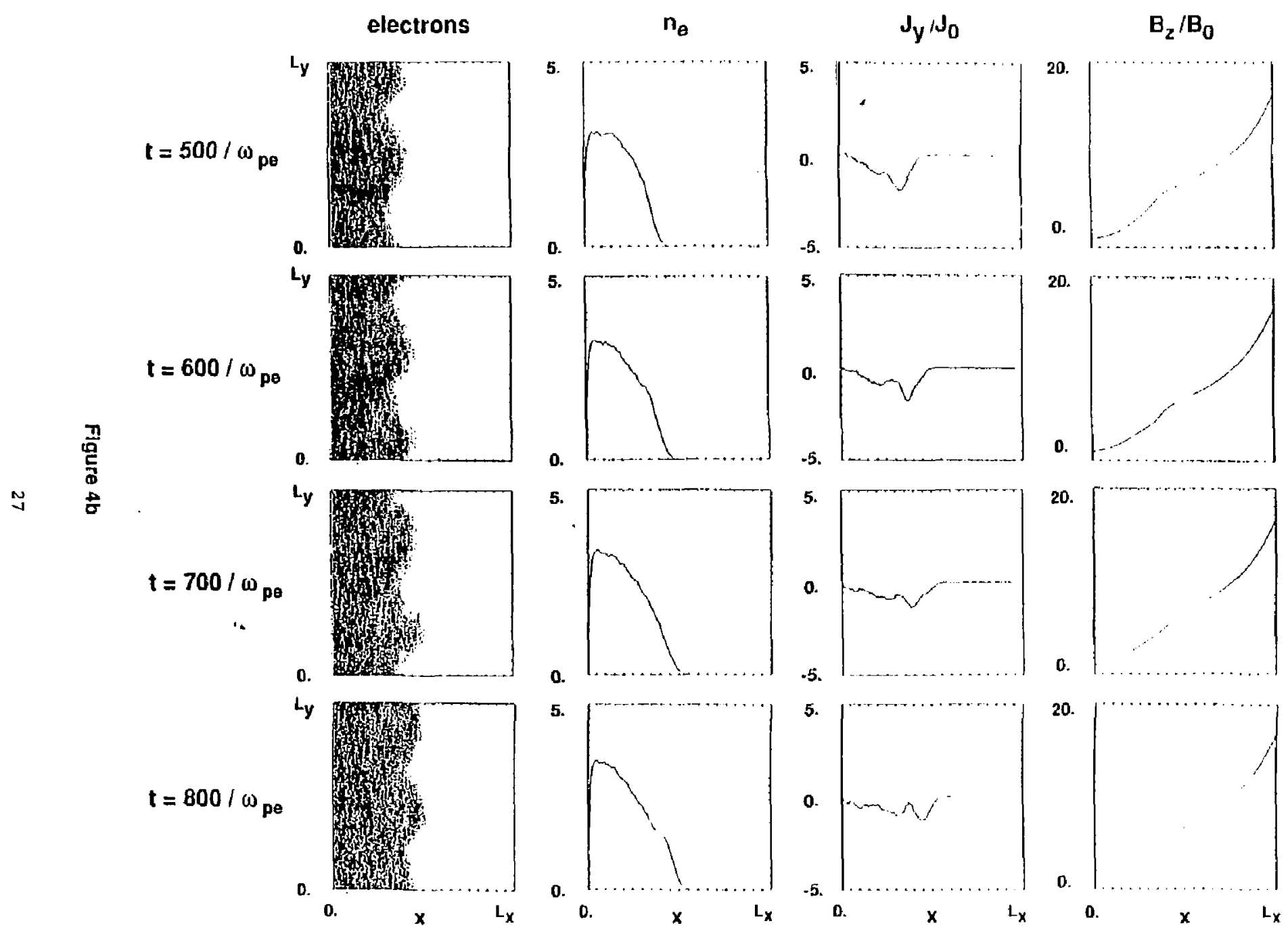

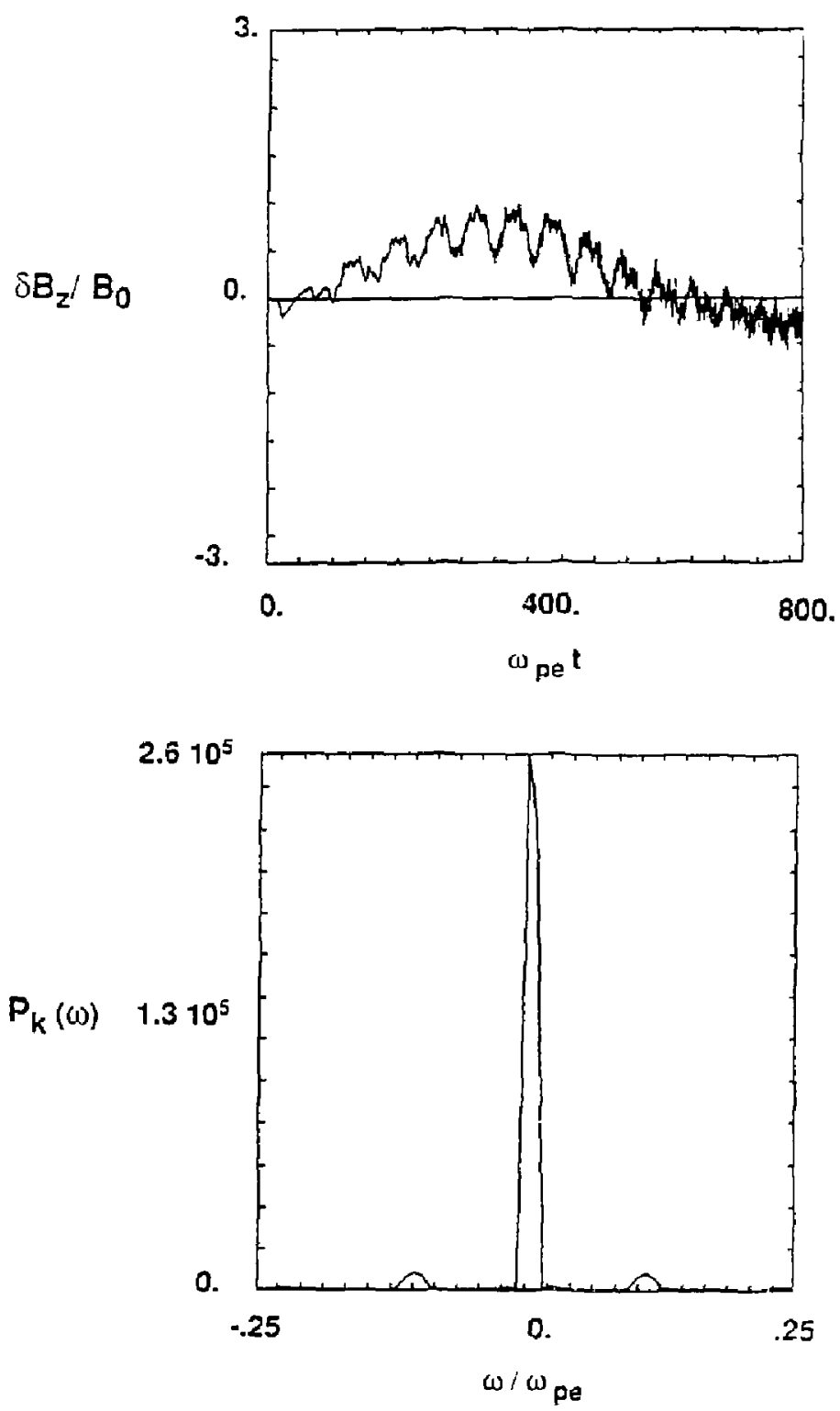

Figure 5: Time history and Power spectrum of $\delta B_{z}\left(x=80 \Delta, k_{y}=0\right)$ 

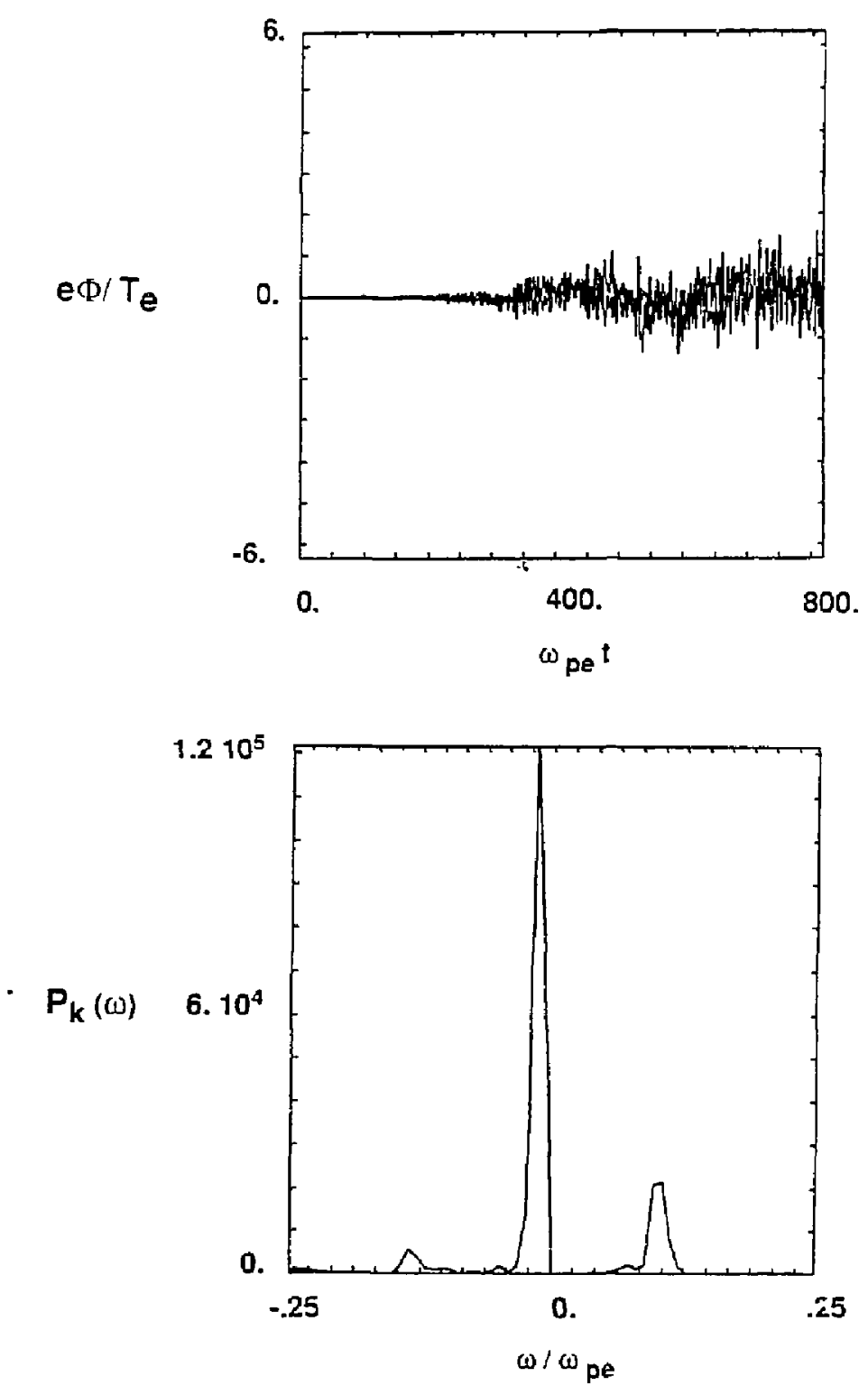

Figure 6a: Time history and Power spectrum of $\Phi\left(x=80 \Delta, k_{y}=4 \pi / L_{y}\right)$ 

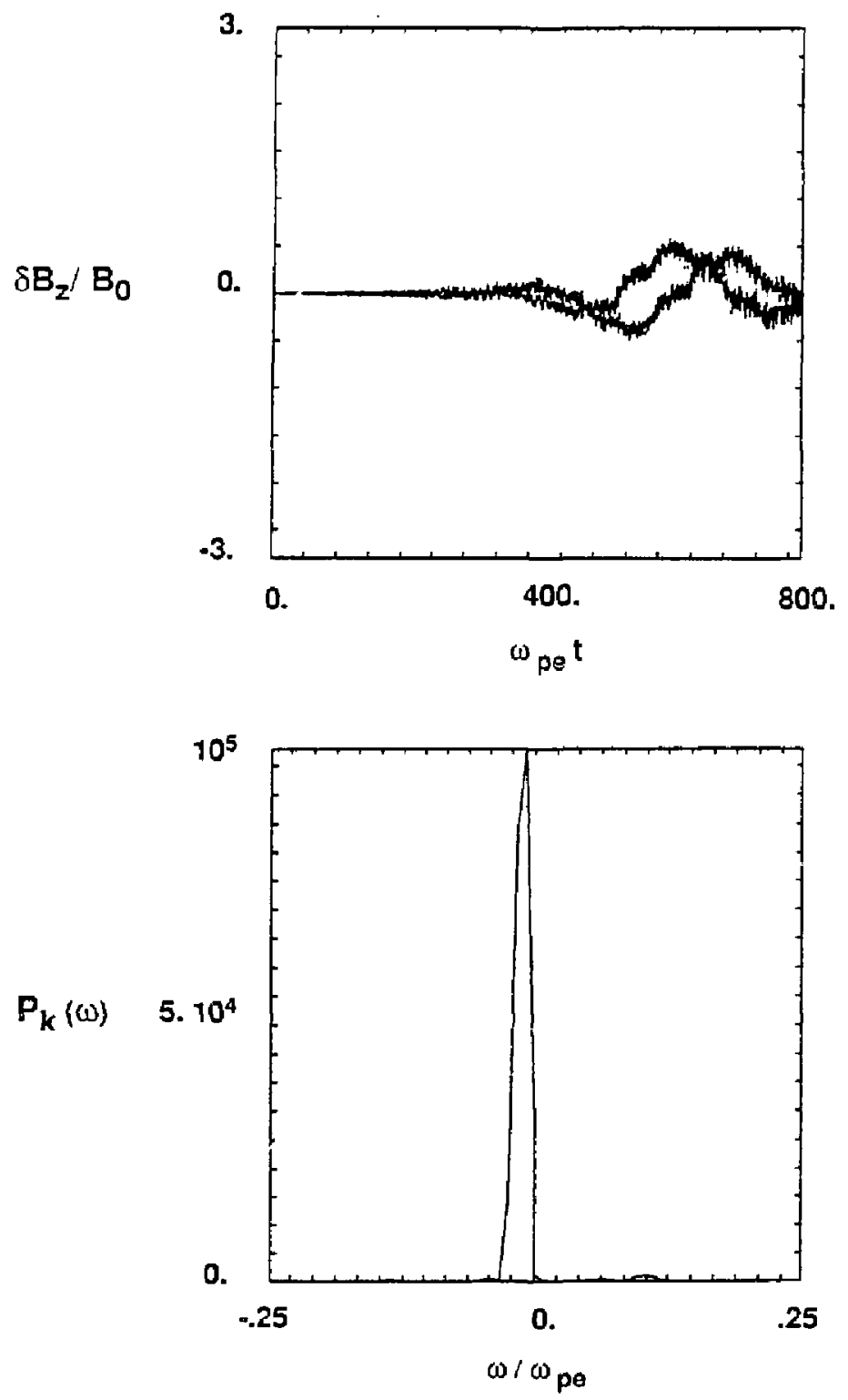

Figure 6b: Time history and Power spectrum of $\delta B_{z}\left(x=80 \Delta, k_{y}=4 \pi / L_{y}\right)$ 


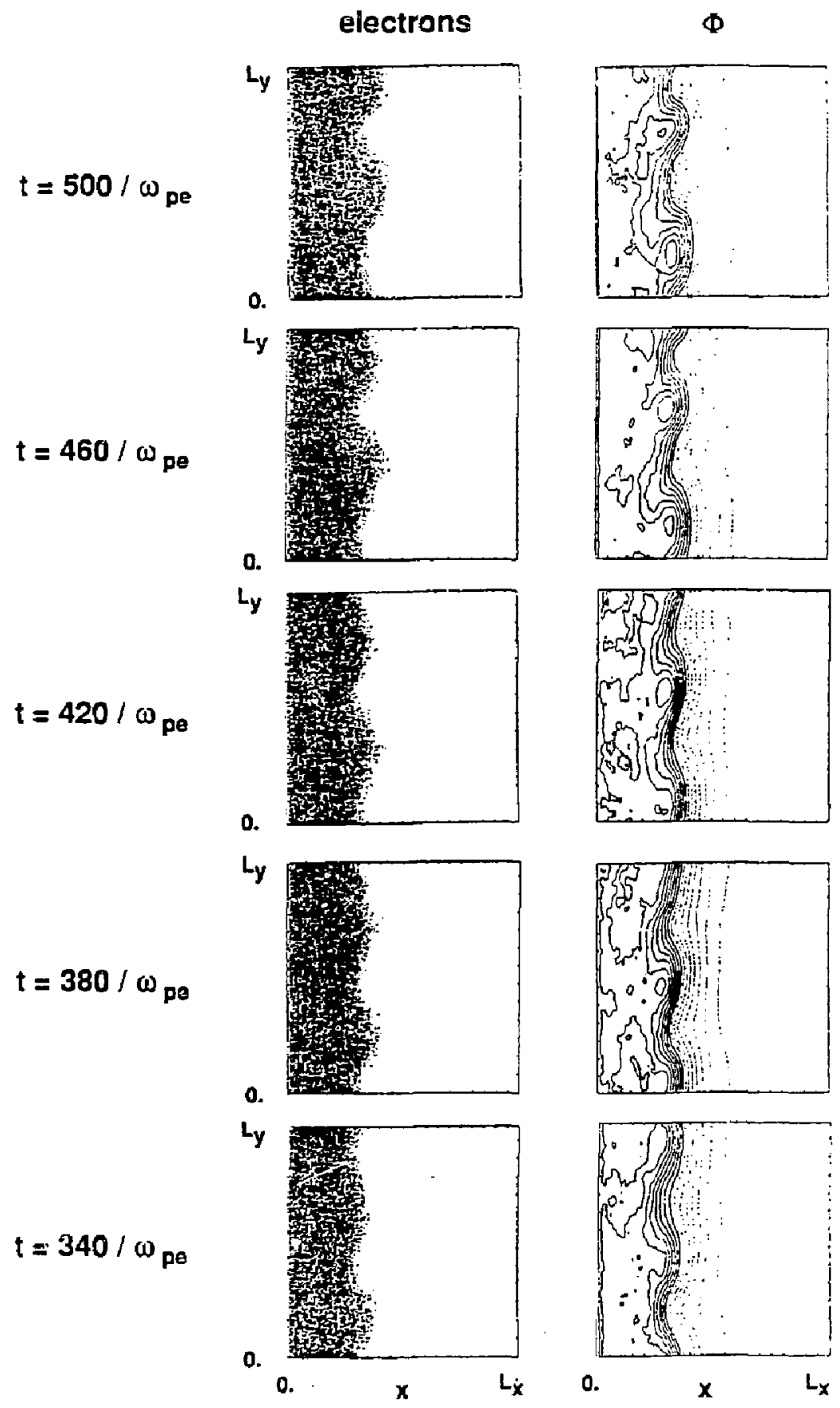

Figure 7 